\title{
Design and Operation of Hall Thruster with Segmented Electrodes
}

\author{
N. J. Fisch, Y. Raitses, A. A. Litvak, and L. A. Dorf \\ Princeton Plasma Physics Laboratory, Princeton University, Princeton NJ 08540
}

\begin{abstract}
Principles of the Hall thruster with segmented electrodes are explored. A suitable vacuum facility was put into service. For purposes of comparison between segmented and conventional thruster approaches, a modular laboratory prototype thruster was designed and built. Under conventional operation, the thruster achieves state-of-the-art efficiencies $(56 \%$ at $300 \mathrm{~V}$ and $890 \mathrm{~W}$ ). Very preliminary results under operation with segmented electrodes are also described.
\end{abstract}

\section{Introduction}

A Hall thruster with segmented emissive electrodes along the thruster channel may have certain advantages over conventional designs, particularly with regard to plume divergence and component lifetime [1,2]. The emissive electrodes can establish very abrupt potential drops in the thruster channel, with electrons entering the channel flow through the electrodes. Dielectric insulators separate the electrodes, with segments held at different potentials through separate power supplies. In the channel, the radial magnetic field provides magnetic insulation. In principle, the efficiency of such thrusters can approach the theoretical maximum. The segmented cathode approach provides a localized acceleration region.

Maximal localizing of the voltage drop accomplishes several things at once. The channel dimensions can be shortened, resulting in decreased weight. The magnetic screen design can be relaxed, also decreasing weight. Most important, the localization can be in a region of concave magnetic field for maximum focusing, resulting in less plume divergence. Less plume divergence results in less erosion and greater ease of system integration.
Flexibly designed laboratory prototypes were built and deployed in the Princeton Plasma Physics Laboratory (PPPL). This paper describes the facility and the preliminary design and operation of segmented cathode thrusters.

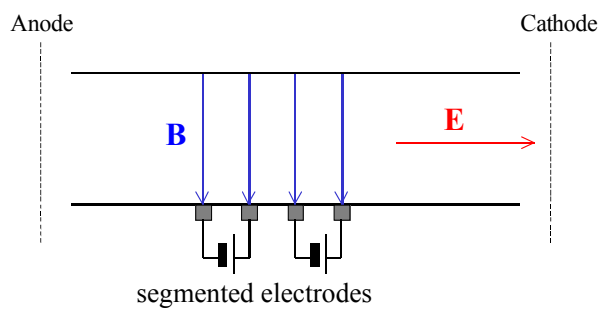

Fig. 1. Segmented electrode Hall Thruster

Like the conventional SPT (Stationary Plasma Thruster) or Hall thrusters, the plasma jet flows in crossed electric and magnetic fields applied in a coaxial channel. The thruster consists of the magnetic circuit, gas distributor (which can also be the anode), insulator channel, cathode-neutralizer and segmented emissive electrodes. The magnetic circuit includes a magnetized source, which can be either an electromagnetic coil or a permanent magnet. The gas propellant enters through the gas distributor and is ionized in the electrical discharge between the anode and the cathode. The applied radial magnetic field impedes the electron flux to the anode, so that the quasi-neutral plasma can 
support a large electric field. This electric field accelerates the ions towards the thruster exhaust. By momentum conservation, the thrust is absorbed by the magnetic circuit. The cathode neutralizer supplies electrons in order to compensate the positive space charge of the emerging ions.

The main difference between the conventional design and the segmented design is that the segmented electrodes give independent control of the potential distribution in the thruster (see Fig. 1). Since the radial magnetic fields form equipotential surfaces, electron emission at the channel surfaces intersecting the magnetic fields can dictate independently the potential of each surface. Separate power supplies control the segmented electrodes, but, in principle, the amount of current drawn, and hence the power, can be very small if the electron mobility is small. However, in this paper, we report only results with a single power supply.

The segmented electrodes provide a way of localizing and controlling the accelerating electric field. This control is key to reducing the beam divergence in the thruster channel. The two main sources of plume divergence are the curvature of the magnetic field and the sheath potential. Note that where the magnetic field lines are purely radial, the electric field is purely axial. Hence, by localizing the potential drop to this region, ions accelerate purely in the axial direction. Also, by providing for a localized voltage drop immediately adjacent to the ionization region, the time spent by ions in the radial electric field associated with the sheath potential can be minimized. Moreover, emissive electrodes will tend to minimize this radial electric field.

\section{Experimental Setup}

\section{Test Facility}

The vacuum system used for the thruster operation experiments consists of a stainless steel vacuum vessel of diameter $2.29 \mathrm{~m}$ and length $8.38 \mathrm{~m}$ (Fig. 2), equipped with 32 inch diffusion pump, and backed by a two stage rootspump system. For xenon gas flow rate of 23 sccm, the pumping speed was $12,0001 / \mathrm{s}$.

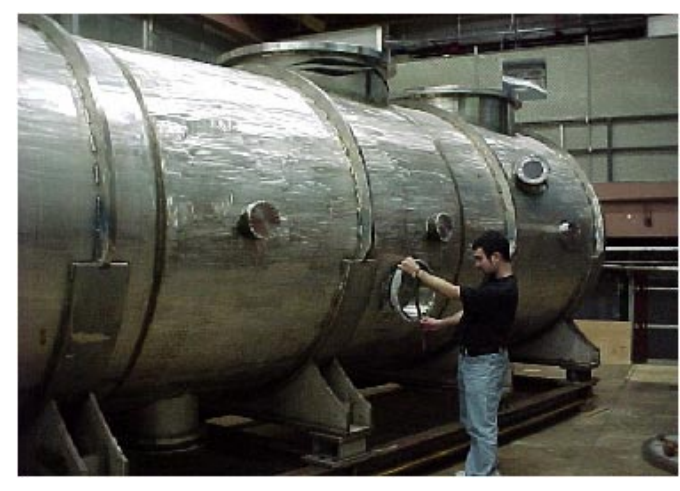

Fig. 2. Vacuum vessel

A commercial mass flow control and measurement system was used to supply xenon to the anode and to the cathode. This system includes two Millipore FC-260 controllers $(0 \div 50$ SCCM for the anode and $0 \div 10$ SCCM for the anode) connected to the LR-250 readout and control box. A $1 \mathrm{kV}$ and $10 \mathrm{~A}$ commercial voltage regulated power supply was used for the main discharge. Additional voltage regulated and current regulated power supplies for the cathode preheating, electromagnetic coil bias and ignition.

\section{Thruster}

To study operation of segmented electrodes, a 1-2 kW laboratory Hall thruster model was designed and built at the PPPL. The thruster view is shown in Fig. 3. The thruster consists of the magnetic circuit, insulator channel, cathode, anode and segmented electrodes. The magnetic circuit of the thruster is made of a low carbon steel and includes a number of replaceable parts in order to allow various modifications of this circuit. Electromagnetic coils, which can be biased by a separate power supply, are used as the magnetized source. The magnetic field inside the thruster was simulated by ANSYS software. The magnetic field was then mapped by using a commercial gaussmeter with miniature Hall probes. Results of magnetic flux simulations and measurements of the magnetic filed along the axis at the median of the thruster channel $(72$ $\mathrm{mm}$ ) at an electromagnetic coils current of $1.5 \mathrm{~A}$, are shown in Fig. 4 and Fig. 5, respectively. 


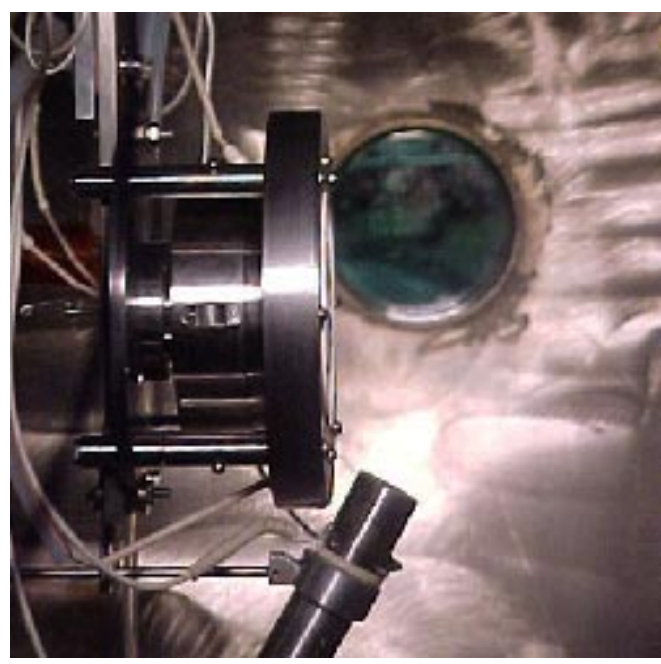

Fig. 3. Laboratory model thruster

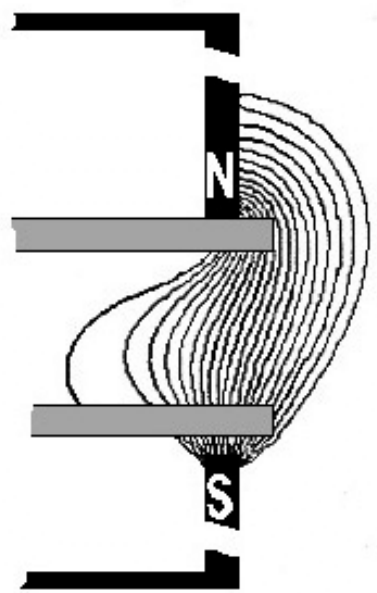

Fig. 4. Simulated magnetic field

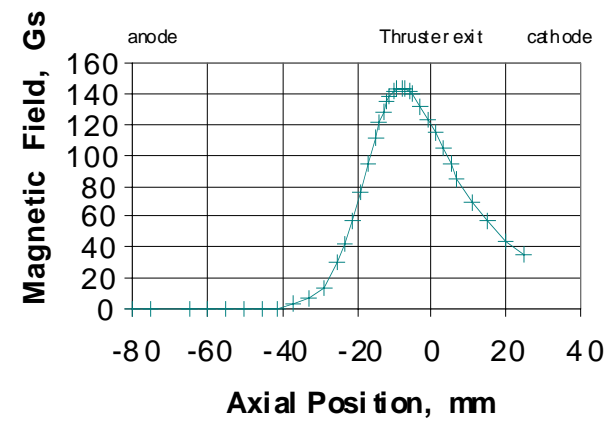

Fig. 5. Measured radial magnetic field magnetic field measured at an electromagnetic coil current of $1.5 \mathrm{~A}$ at the median of the laboratory thruster.

A $90 \mathrm{~mm}$ diameter thruster channel was made from boron nitride ceramic. A flexible construction permits segmented electrodes to be placed either on the inner or the outer wall. Since the anode serves also as a gas distributor, it is constructed so as to provide a uniform gas injection to the annular channel. The length between the anode and the thruster exit is about $50 \mathrm{~mm}$. A hollow-type cathode-neutralizer with $\mathrm{LaB}_{6}$ emitter was developed for the thruster experiments. In addition, we used also a commercial HeatWave hollow cathode.

A set of non-emissive segmented electrodes was manufactured for the purpose of this preliminary study. One electrode was positioned on the cathode-side of the magnetic field maximum (cathode-side electrode), while the other electrode was positioned to the anode side of the field maximum (anode-side electrode). The anode-side electrode was made from stainless steel, while the cathode-side electrode was made from tantalum in order to reduce sputtering.

\section{Diagnostic Setup}

To measure the thrust, a pendulum-type thrust stand was designed and built (Fig. 6). The thruster was suspended on the arm, connected to a high-sensitivity inclinometer, so that accurate measurements could be made of the arm deflection angle, which measures the thrust. The thrust stand calibration system consists of an arrangement of weights, $0.1-5 \mathrm{~g}$, applied to the pendulum through a pulley. The thrust stand calibration experiments were performed with and without thruster operation. They demonstrated good linearity with high reproducibility. Although the thrust resolution of this thrust stand is $1 \mathrm{mN}$ (minimal calibrating weight applied), lower values of thrust were detectable.

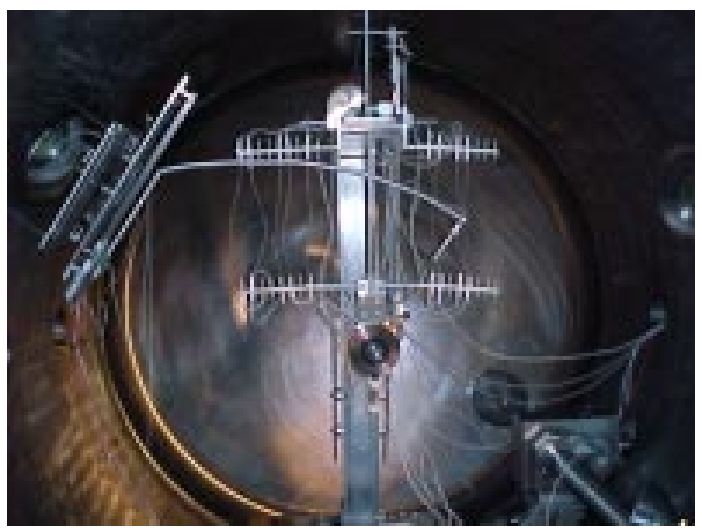

Fig. 6. Thrust stand and probe setup 
In addition to the thrust, propellant mass flow and discharge characteristics, the angular ion current distribution was measured using a flat Langmuir probe. The probe is a circular disk made of low-sputtering tungsten-copper alloy with a collecting area of $2.83 \mathrm{~cm}^{2}$. The probe is mounted on a rotating arm, which is assembled on a positioning setup (Fig.7). This setup allows a probe motion on a circle with the center on the thruster axis. The distance between the probe and the thruster can be varied from $0.1 \mathrm{~m}$ to $1 \mathrm{~m}$. During thruster operation, the probe is moved around the thruster at a certain radius, while its angular position is measured using a linear highresolution potentiometer. A negative bias voltage is applied to the probe from a voltageregulated power supply. The probe current is determined by voltage measurements on a $100 \Omega$ shunt resistor. Assuming axially symmetric ion flux distribution, the collected probe current data is interpolated and integrated, yielding the total ion current at the thruster exhaust.

\section{Experimental Procedures}

The thruster operation was investigated for three different configurations: without segmented electrodes, with one positive segmented electrode, and with two segmented electrodes (positive and negative). In addition, in two different sets of experiments, two negatively biased segmented electrodes of thickness $0.1 \mathrm{~mm}$ and $4 \mathrm{~mm}$ were used. The thruster measurements were performed with xenon gas flow rate in the range of $1-3.3 \mathrm{mg} / \mathrm{s}$ and a discharge voltage of 100-300 V. In addition, the electromagnetic coil current was varied.

In the first set of experiments, the thruster was operated without segmented electrodes. In each operating point, which is defined by the discharge voltage, gas flow rate and electromagnetic coils current, the thruster discharge characteristics, plume and thrust measurements were taken, when the thruster, thrust stand and probe reached a steady state. In order to verify reproducibility of these measurements, operating points at a given discharge voltage and at each gas flow rate were repeated at the end of each thruster run. Generally speaking, if there were observable differences, they were within the thrust resolution $(1 \mathrm{mN})$.

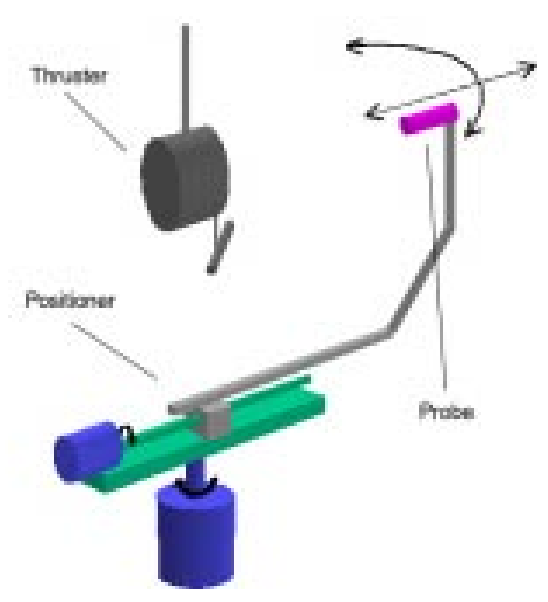

Fig. 7. Ion current distribution measurement

For each operating point, specific impulse, $I s p=T / m$, and thruster efficiency, $\eta=T^{2} / m P_{e}$, were deduced from thrust, $T$, mass flow rate, $m$, and input power measurements, $P_{e}$. In addition, these estimates were corrected by taking into account an additional xenon gas flow rate affected by background pressure [3]. In these experiments, this additional mass flow rate was not larger than $1 \%$ of the measured gas flow rate through the thruster. The uncertainties in the determination of the thruster performance were estimated as static RMS error [3].

The probe characteristics were measured at a number of different thruster operating points and probe positions. In all these measurements, a saturation of the ion current to the probe was clearly observed at a bias voltage of $-30 \mathrm{~V}$. Therefore, all measurements of the ion angular distribution were performed at this bias voltage. Although the total ion current is uncertain from these measurements, relative measurements for comparison between different thruster configurations can be made. All probe measurements presented here were performed at the distance of $37 \mathrm{~cm}$ from the thruster exit.

This procedure of thruster measurements and determination of their errors was also applied in experiments with the other thruster configurations. All these experiments took about 60 hours of thruster operation. 


\section{Results and discussions}

To focus on the physical processes inside the thruster channel, the mass flow rate through the cathode was held constant, about $0.3 \mathrm{mg} / \mathrm{sec}$ at all operating points. Thus, the thruster performance was estimated without taking into account the flow rate through the cathode. All values of the mass flow rate here represent the flow rate through the anode corrected for the background pressure effect.

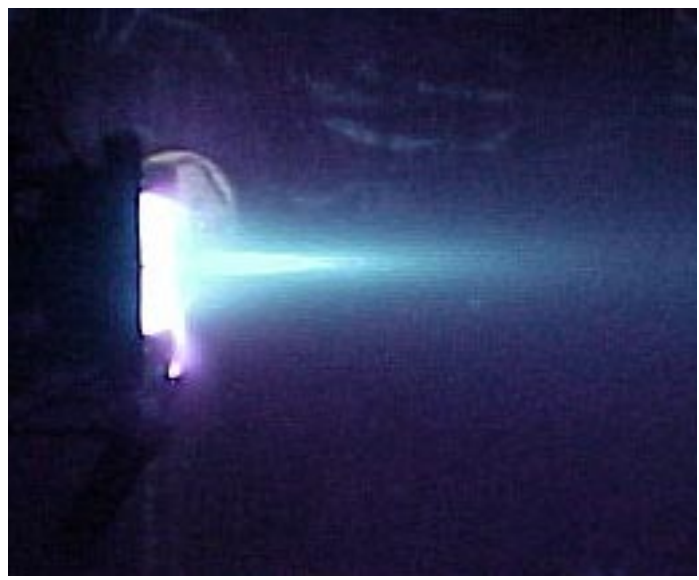

Fig. 8. Hall thruster operation

\section{Operation without segmented electrodes}

To compare the operation of segmented electrode Hall thruster with its conventional counterpart, the discharge characteristics and performance of the laboratory thruster were measured without segmented electrodes under various operating conditions. Fig. 9 shows the discharge current versus the electromagnetic coil current at a constant discharge voltage of $250 \mathrm{~V}$. Fig. 10 shows the V-I characteristics. These measurements are made for the following mass flow rates: $1.7 \mathrm{mg} / \mathrm{s}, 2 \mathrm{mg} / \mathrm{s}, 2.5 \mathrm{mg} / \mathrm{s}$ and 3 $\mathrm{mg} / \mathrm{s}$. As expected, the non-segmented thruster operation closely resembles typical behavior of Hall thrusters [3, 4]. For example, as can be seen in Fig. 9, for each of the presented curves, there is so-called "optimal" value of the electromagnetic coil current, at which point the discharge current reaches its minimum. It was observed that this value increases with the gas flow rate and the discharge voltage.

An increase of the discharge current above this optimal value of the coil current is typically attributed to large amplitude discharge oscillations together with an increase of the electron mobility across the magnetic field [5]. Although the physical phenomena, behind this behavior, is not clear, it may indicate a limit to the magnetic insulation in Hall thrusters, possibly related to a plasma instability. Since the thruster efficiency is obviously maximized when the magnetic insulation is maximized, we describe in the following operating points at this "optimal value" of the electromagnetic coils current.

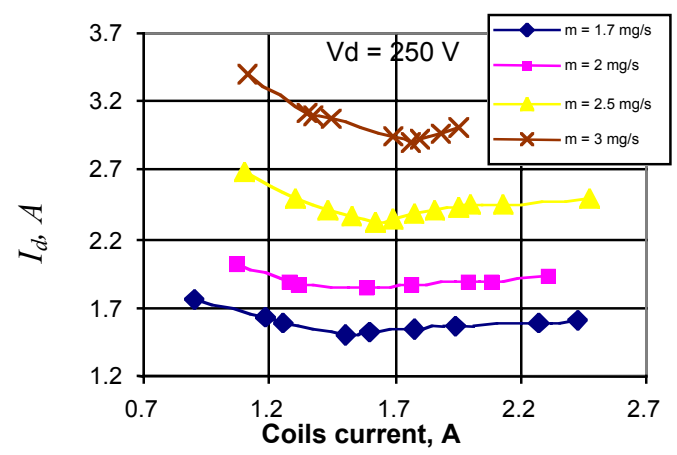

Fig. 9. Discharge current as function of coil current, without segmented electrodes, for gas flow rates: 1.7, $2.0,2.5$ and $3 \mathrm{mg} / \mathrm{s}$, at discharge voltage of $250 \mathrm{~V}$.

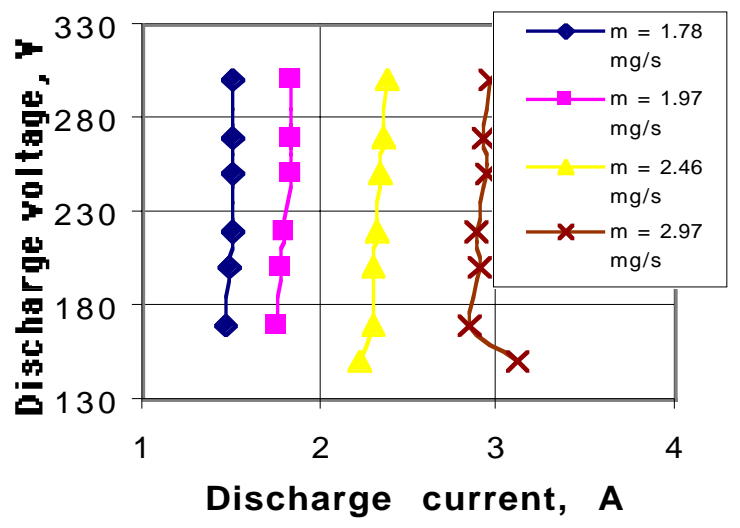

Fig. 10. V-I characteristics without segmented electrodes, at gas flow rates: 1.7, 2.0, 2.5, and 3.0 $\mathrm{mg} / \mathrm{s}$.

The measured $V_{d}-I_{d}$ characteristics also demonstrate typical behavior of the electrical discharge in Hall thrusters [4]. As can be seen in Fig. 10, at each of four mass flow rates, an increase of the discharge voltage hardly changes the discharge current, at least at discharge voltages larger than $200 \mathrm{~V}$. In addition, the measurement of the ion angular distribution, discussed below, indicated that changes in the total ion current, $I_{i}$, are also insignificant as the 
discharge voltage at a given gas flow rate. Thus, in these operating regimes, the propellant utilization, $I_{i} M_{i} / \mathrm{em}$ and the electron mobility across the magnetic field, which can be derived from the ratio of $\mathrm{I}_{\mathrm{i}} / \mathrm{I}_{\mathrm{d}}$, are hardly affected by the discharge voltage at a given mass flow rate.

The behavior of the thruster performance versus the mass flow rate, measured for the laboratory thruster without segmented electrodes at three values of the discharge voltage (200, 250 and $300 \mathrm{~V}$ ) is shown in Fig. 11. At a given discharge voltage, the thrust (Fig. 11a) increases almost linearly when the gas flow rate is varied from $1.7 \mathrm{mg} / \mathrm{s}$ to $3.0 \mathrm{mg} / \mathrm{s}$. This is obviously mainly due to the increase of the ion current with the gas flow rate.

Note that the laboratory thruster without segmented electrodes demonstrates performance similar to that of state-of-the-art Hall thrusters, making more meaningful comparison with the segmented modifications. For example, at input power of $890 \mathrm{~W}(300 \mathrm{~V}$ of the discharge voltage, $3 \mathrm{mg} / \mathrm{s}$ of the anode mass flow rate) the thruster achieves specific impulse of 1856 s (1690 with the cathode flow rate) and efficiency about $56 \%$ ( $51 \%$ with the cathode flow rate). A preliminary analysis of thruster operation with segmented electrodes is described below.

\section{One anode-side segmented electrode}

The effect of a segmented electrode, placed on the anode side of the channel relative to the magnetic poles was studied. In the experiments described below, this electrode was not biased and the distance between the median of this electrode and the thruster exit was $10 \mathrm{~mm}$.

Figs. 12 and 13 exhibit typical behavior of the ion current at the thruster exit versus the discharge voltage and the ratio of the ion current to the discharge current measured for three mass flow rates: $1.7 \mathrm{mg} / \mathrm{s}, 2 \mathrm{mg} / \mathrm{s}$ and $2.5 \mathrm{mg} / \mathrm{s}$. In addition, the thrust, specific impulse and the efficiency versus the discharge voltage for the same mass flow rates are shown in Fig. 14. As can be seen from Figs. 12 and 13, for most of operating points measured with the nonsegmented thruster configuration, the ion current and the current ratio are slightly larger than for the one-segmented configuration of the thruster.

However, differences in the measured behavior of the thrust and thruster performance are even smaller for these two cases, at least at discharge voltages larger than $200 \mathrm{~V}$. Apparently, under these circumstances, the thruster operation was hardly affected by the insertion of the segmented electrode into the channel.

a)

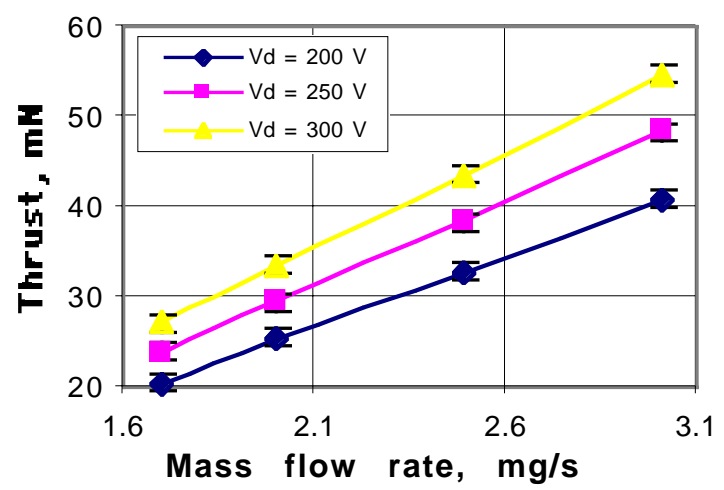

b)

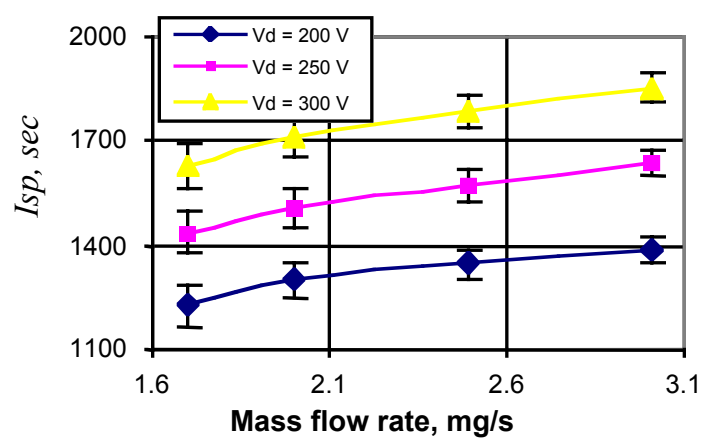

c)

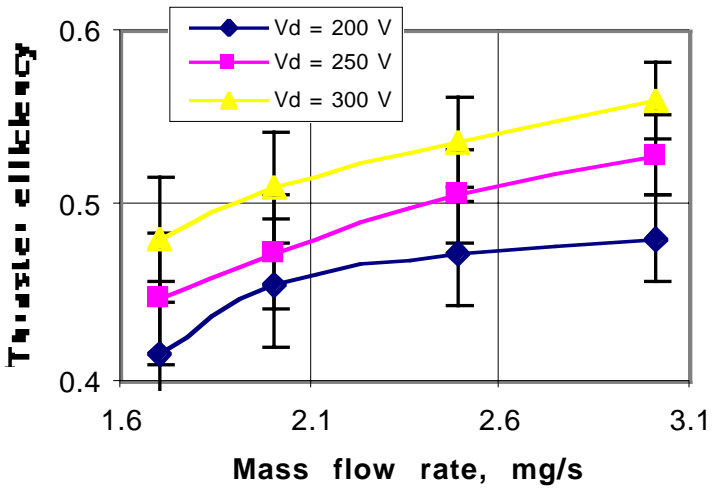

Fig. 11. Thrust, specific impulse, and thruster efficiency versus mass flow rate without segmented electrodes for discharge voltages, $200 \mathrm{~V}, 250 \mathrm{~V}$ and $300 \mathrm{~V}$. 


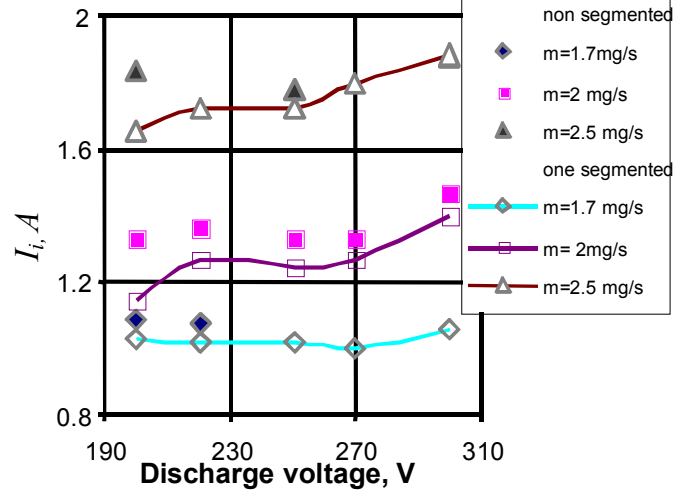

Fig. 12. Ion current at thruster exit versus discharge voltage at mass flow rates: $1.7,2.0$ and $2.5 \mathrm{mg} / \mathrm{s}$, either without segmented electrode or with one anodeside segmented electrode.

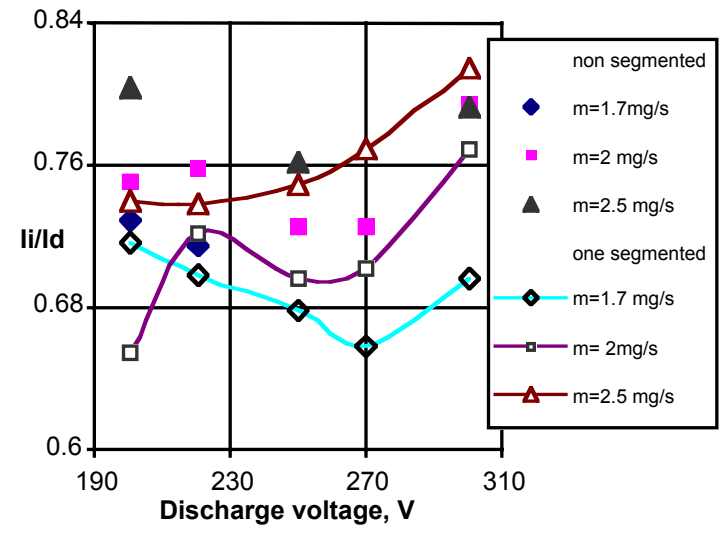

Fig. 13. Ratio of ion current at thruster exit to discharge current versus the discharge voltage at mass flow rates: $1.7,2.0$ and $2.5 \mathrm{mg} / \mathrm{s}$, either without segmented electrode or with one anode-side segmented electrode. a)

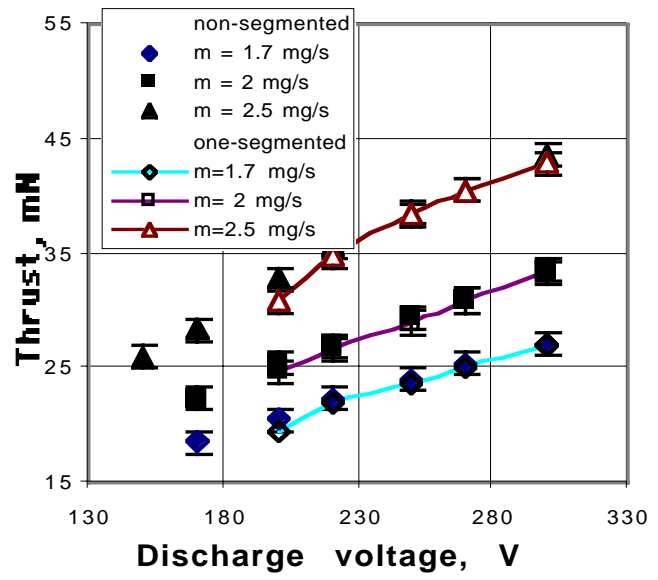

b)

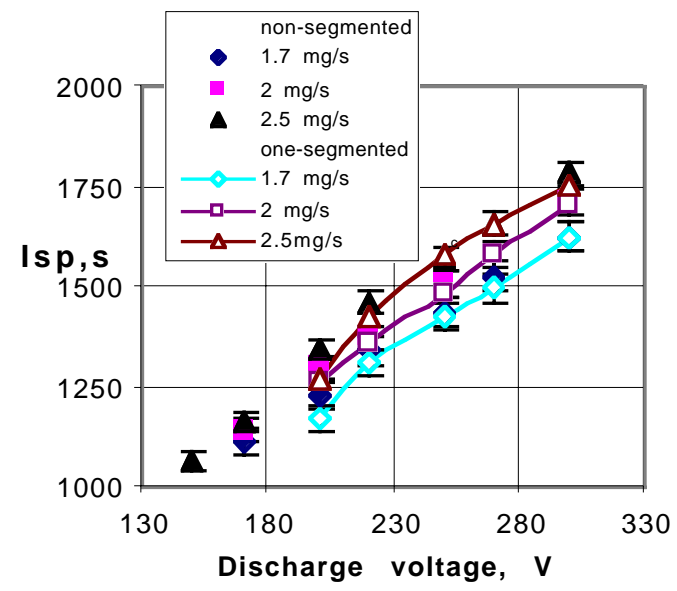

c)

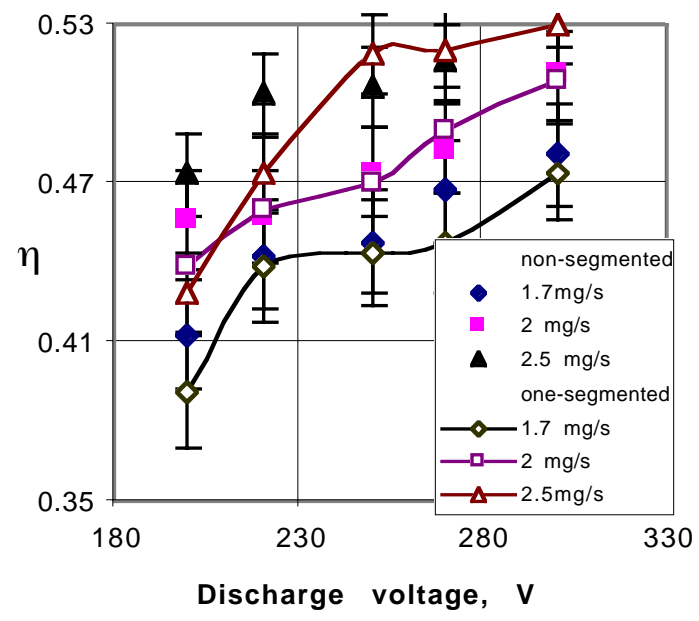

Fig. 14. Thrust, specific impulse, and thruster efficiency versus discharge voltage for various mass flow rates, either without segmented electrode or with one anode-side segmented electrode. 


\section{Two segmented electrodes}

The measurements below describe the thruster operation with one anode-side segmented electrode and two cathode-side segmented electrodes, either thin $(0.1 \mathrm{~mm})$ or thick $(4 \mathrm{~mm})$. Results are compared with those obtained with non-segmented and one segmented configurations of the laboratory thruster. Different sets of measurements were done with thick and thin cathode-side electrodes. Each cathode-side electrode was under the cathode potential, while the anode-side electrode was under a floating potential.

Fig. 15 illustrates the behavior of the floating potential of the unbiased anode-side electrode, which was measured relative to the anode potential, for the two biased cathode-side electrode cases: thick and thin, respectively. Measurements were made at mass flow rates of 1.7 and $2.0 \mathrm{mg} / \mathrm{s}$.

It is rather startling that the floating potential relative to the anode for each segmented electrode case is smaller than in the case where the cathode-side electrode is absent. In the absence of the plasma, the vacuum voltage drop would suggest just the opposite. Here, a greater voltage potential drop is then established between the anode-side electrode and the thruster exit (where the negatively-biased segmented electrode is located).

For a thick cathode-side segmented electrode, this phenomenon persists as the discharge voltage increases (Fig. 15b). For the thin segmented electrode case, however, this phenomenon diminishes with the discharge voltage (Fig. 15a).

It is also quite remarkable that when the cathode-side electrode was unbiased, the floating potential of the anode-side electrode relative to the anode was almost unchanged from the biased case. For example, at mass flow rate of $1.7 \mathrm{mg} / \mathrm{s}$ and discharge voltage of $220 \mathrm{~V}$, the floating potential of the anode-side electrode was $11.3 \mathrm{~V}$ in the biased case and $12.2 \mathrm{mg} / \mathrm{s}$, when the cathode-side segmented electrode was unbiased, and floating at a potential of $52.3 \mathrm{~V}$. From all these measurements of the floating potential, it is not clear if the length of acceleration and ionization regions was also affected by the biasing of the segmented electrode. Possibly, there is some sheath phenomena, which may be appreciable, because the electron temperature in Hall thrusters, typically $10-20 \mathrm{eV}$, is close to the magnitude of the observed changes in the floating potential. For better understanding of this behavior, direct measurements of the voltage potential distribution in the thruster channel are obviously necessary.

a)

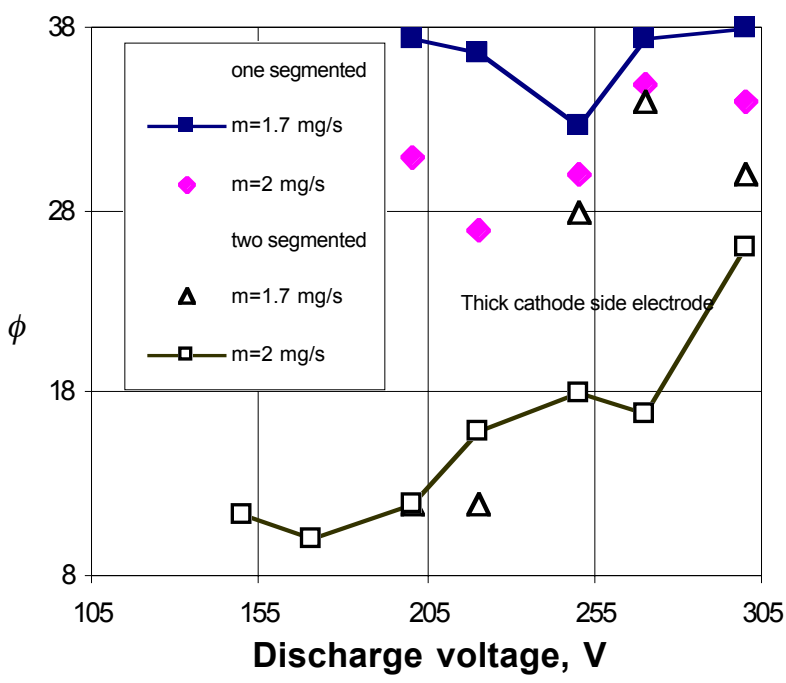

b)

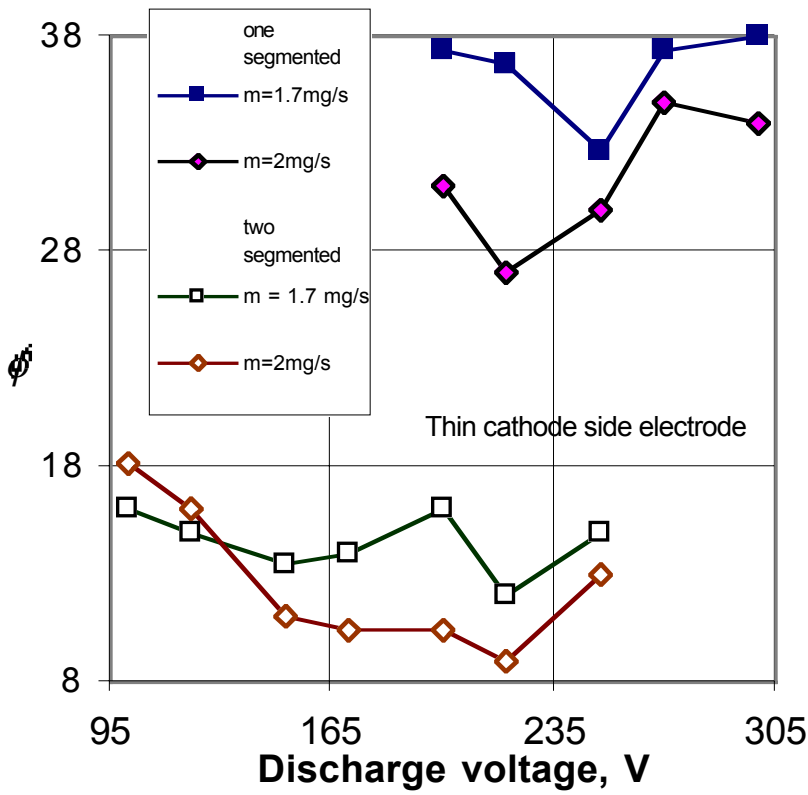

Fig. 15. Anode-side segmented electrode floating potential, $\phi$, relative to anode versus discharge voltage, with cathode-side segmented electrodes: a) thick (4 mm); and b) thin $(0.1 \mathrm{~mm})$. 

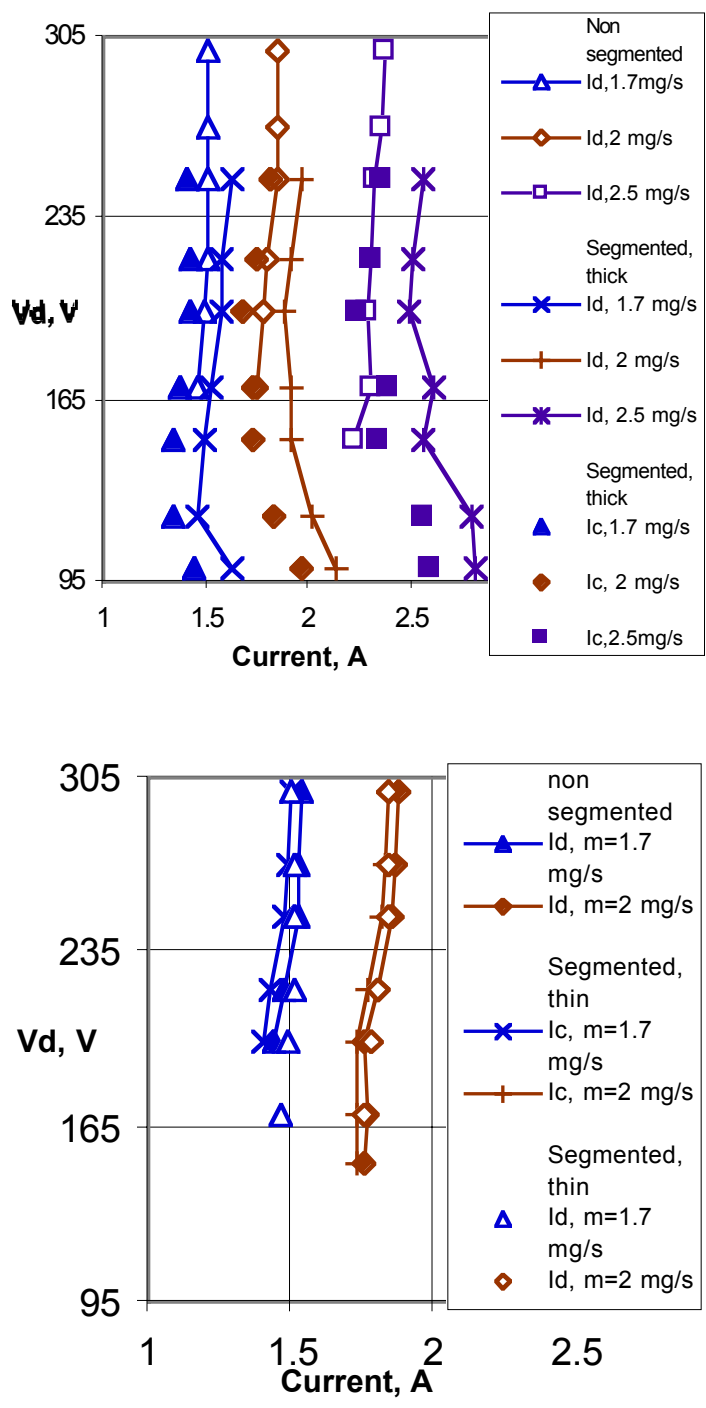

Fig. 16. $\quad \mathrm{V}-\mathrm{I}_{\mathrm{c}}$ characteristics for cathode-side segmented electrodes, a) thick and b) thin, with anode-side floating segmented electrode, and at different mass flow rates.

The I-V characteristics for the thick electrode with mass flow rates, 1.7, 2.0, and 2.5 $\mathrm{mg} / \mathrm{s}$, and for the thin segmented electrode, with 1.7 and $2.0 \mathrm{mg} / \mathrm{s}$, are shown in Figs. $16 \mathrm{a}$ and $\mathrm{b}$ respectively. The cathode current in the segmented cases was estimated by subtracting the current measured in the circuit of the cathode-side segmented electrode from the total current of the power supply. In the nonsegmented configuration, this total current is equal to the discharge current

Note that the discharge current measured with the two segmented configurations is generally larger than that obtained for non segmented case. This is due to the presence of the current in the circuit of these segmented electrodes.

More interestingly, note that the compensating cathode current is smaller in the case of the biased cathode-side segmented electrodes, but only for the smaller mass flow rates. Possibly, this is caused by ion collection on the segmented electrode, rather than electron emission. Thermionic electron emission from the surface of the negatively-biased segmented electrodes is likely negligible due to their low temperature. Yet, this current anomaly was measured also in a cold thruster immediately after start-up. Also, since the electron density and electron temperature are typically small at the thruster exit, while most of the potential drop takes place upstream of this electrode, field emission is likely insignificant.

Fig. 17 shows the measured ion current at the thruster exit versus the discharge voltage for non segmented and segmented thick and thin thruster configuration cases. Fig. 18 shows the ratio of the ion current to the cathode current for the thick electrode case. The ion current measured for the segmented configurations is, in general, smaller than that for the non segmented configuration. In addition, at a mass flow rate of $1.7 \mathrm{mg} / \mathrm{s}$ and at discharge voltage below $250 \mathrm{~V}$, the difference between the ion current measured for the thick segmented and for the nonsegmented cases is almost equal to the difference between the cathode current and the discharge current for each of these cases.

For example, for the thick segmented case, at the discharge voltage of $200 \mathrm{~V}$ and a mass flow rate of $1.7 \mathrm{mg} / \mathrm{s}$, the ion current, discharge current and the cathode current are about $1 \mathrm{~A}$, 1.6 $\mathrm{A}$ and $1.42 \mathrm{~A}$, respectively. For the non segmented configuration, $I_{I}=1.1$ A and $I_{d}=I_{c}=1.5$ A. Thus, at this small mass flow rate, the difference in the behavior of the thruster with segmented and non-segmented configurations is probably mainly due to a collection of ions by the cathode-side segmented electrode.

However, interestingly, as the mass flow rate is increased, the ratio of the current collected on the thick segmented electrode to the discharge current decreases, while the ratio of the ion current to the discharge current also decreases. This means that the difference between the ion and total current can not be explained only by the effect of ion collection on the segmented electrode. It is noteworthy that similar behavior is also found for smaller mass flow rate at higher discharge voltages. 
The ratios of the ion current to the total current of the power supply and to the cathode current for the thick segmented case versus the discharge voltage are shown in Fig. 18. The current ratios with the segmented electrodes are generally smaller than that obtained with the non-segmented electrode configuration. In addition, for the segmented case, the ratio of the ion current to the cathode current is larger for the small mass flow rate of $1.7 \mathrm{mg} / \mathrm{s}$ than for $2 \mathrm{mg} / \mathrm{s}$, at least for discharge voltages smaller than 250 V.

Although this behavior is not understood at the moment, a tentative explanation is as follows. The current from the cathode supplies electrons both for neutralization of the emerging ion beam from the thruster and in order to sustain the electrical discharge between the anode and cathode. Since the ion flux from the thruster was smaller in the segmented case, a degradation of the ratio, $\mathrm{I}_{\mathrm{I}} / \mathrm{I}_{\mathrm{c}}$, is due to an increase of the electron current into the thruster from the cathode. This increase of the electron current from the cathode may indicate that the electron mobility across the radial magnetic field is also increased in the channel with the segmented electrodes, in particular, at large mass flow rates. The increased mobility may be due to an interaction between the plasma and the segmented electrodes or due to poor ionization in the region between the anode and positive segmented electrode, which would imply an increase of electron-atom collisions in this gap.

Fig. 19 compares the thruster performance measured for the segmented (thick) and nonsegmented configurations of the laboratory thruster versus the discharge voltage. For the specific parameters considered here, the efficiency of the segmented case is somewhat lower than for the non segmented case.

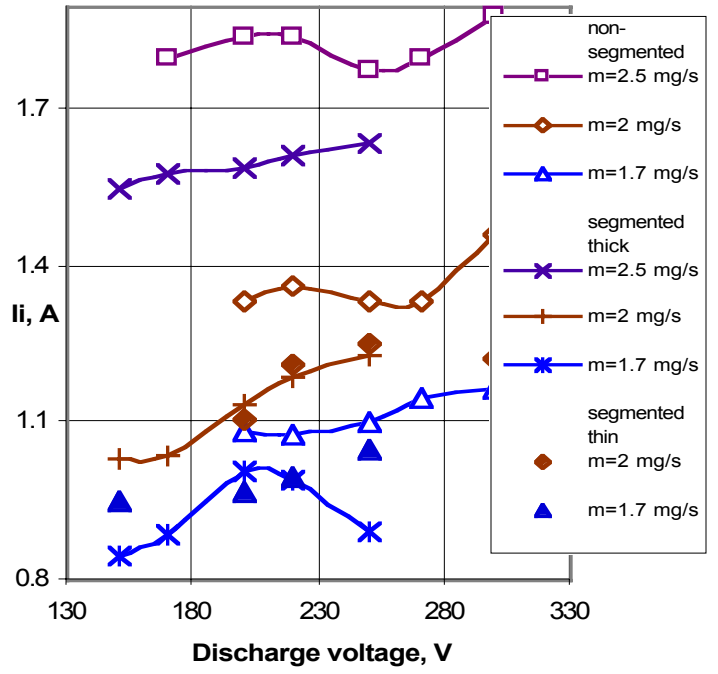

Fig. 17. Ion current versus discharge voltage for nonsegmented and thin and thick cathode-side segmented electrodes for mass flow rates $1.7,2.0$, and $2.5 \mathrm{mg} / \mathrm{s}$.

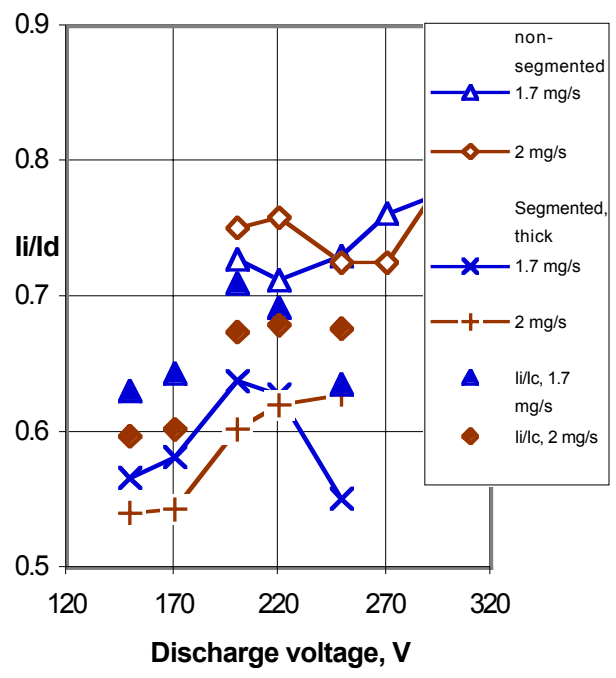

Fig. 18. Ratio of ion current $I_{I}$ to discharge current $I_{d}$ and to cathode current $I_{c}$ versus discharge voltage for non-segmented, and thin and thick cathode-side segmented electrodes, for mass flow rates 1.7 and $2.0 \mathrm{mg} / \mathrm{s}$. 


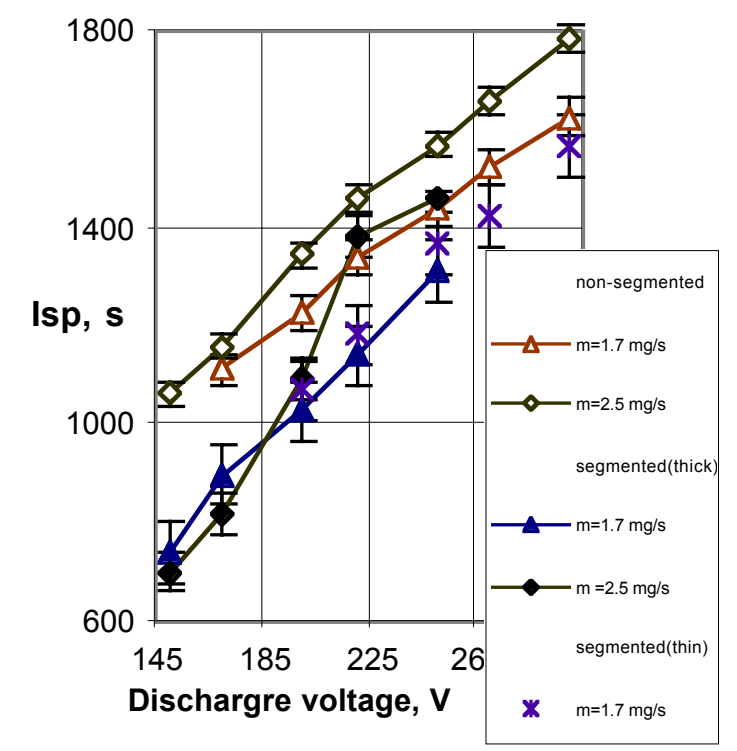

c)

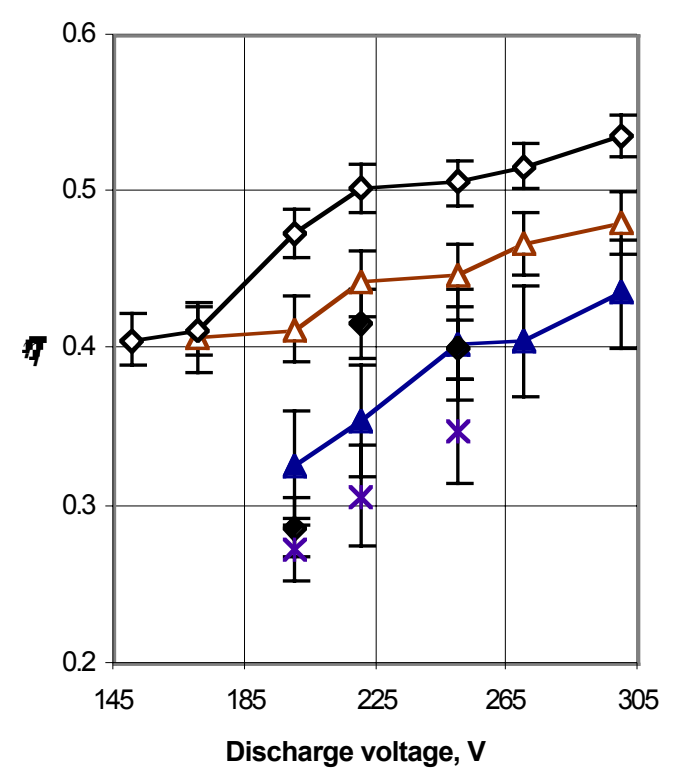

Fig. 19. Specific impulse and thruster efficiency versus discharge voltage at two mass flow rates for different segmented thruster configurations.

\section{Conclusions}

The laboratory thruster, experimental setup, diagnostics, and preliminary results of operation and measurements on a segmented electrode Hall thruster are described. The investigation of the thruster integral characteristics and plume were performed over a broad range of the thruster input parameters with different thruster configurations. The thruster with the non- segmented electrode configuration demonstrated performance comparable to state-of-the-art Hall thrusters. A comparison of the thruster operation with and without segmented electrodes indicated a number of issues associated with the use of non-emissive segmented electrodes, namely, ion losses, ionization efficiency, and possible enhanced electron mobility across the short gap between the segmented electrodes. These issues likely contributed to a somewhat lower thruster performance of the segmented configuration.

The very preliminary results reported here showed that the physical processes involved in a Hall thruster, even with only one or two segmented electrodes, are quite complicated and exhibit unusual and curious behavior. Further theoretical and experimental efforts are indicated and in progress.

\section{Acknowledgment}

The authors thank R. Yager and G. Rose for their excellent technical assistance. The authors also gratefully acknowledge useful discussions with Professor Amnon Fruchtman and Professor Edgar Choueiri.

\section{References}

1. Morozov, A. I., "The Study of Plasma Systems with Closed Electron Drift and Distributed Electric Field," in Plasma Accelerators (ed. L. Artsimovich), Moscow, Mashinostroenie (1973). (in Russian)

2. Fruchtman, A., Fisch, N. J., "Modeling the Hall Thruster," AIAA 98-3500, Cleveland, Ohio (1998).

3. Brophy, J. R., "Stationary Plasma Thruster Evaluation in Russia," Jet Propulsion Lab., JPL-92-4, Pasadena, CA (March, 1992).

4. Bugrova, A. I., Kim, V., Maslennikov, N. A., and Morozov, A. I., "Physical Processes and Characteristics of Stationary Plasma Thrusters with Closed Electron Drift," $22^{\text {nd }}$ Int. Electric Propulsion Conf., IEPC-91-79, Viareggio, Italy (Oct. 1991).

5. Raitses, Y., Ashkenazy, J., "Discharge Characteristics of Hall Current Accelerators," Proc. XVII Int. Symposium on Discharges and Electrical Insulation in Vacuum, Berkeley, CA (July, 1996). 